\title{
Vera Bakšytė, langue et survie d'un peuple
}

\section{Philippe Edel}

\section{(2) OpenEdition}

Journals

Édition électronique

URL : http://journals.openedition.org/rbnu/691

DOI : 10.4000/rbnu.691

ISSN : 2679-6104

\section{Éditeur}

Bibliothèque nationale et universitaire de Strasbourg

\section{Édition imprimée}

Date de publication : 1 novembre 2017

Pagination : 72-75

ISBN : 9782859230678

ISSN : 2109-2761

\section{Référence électronique}

Philippe Edel, «Vera Bakšytė, langue et survie d'un peuple », La Revue de la BNU [En ligne], 16 | 2017, mis en ligne le 01 juillet 2019, consulté le 28 décembre 2020. URL : http://journals.openedition.org/ rbnu/691; DOI : https://doi.org/10.4000/rbnu.691

\section{(C) $10(0$}

La Revue de la BNU est mise à disposition selon les termes de la Licence Creative Commons Attribution - Pas d'Utilisation Commerciale - Partage dans les Mêmes Conditions 4.0 International. 


\title{
Beitray zur Geschichte des Kampfes \\ um die Schulsprache in Litauen
}

\author{
mit besonderer Berücksichtigung \\ der Zeit der Großen Reformen (1855-1864)
}

s

\author{
Inaugural-Dissertation \\ zur Erlangung der philosophischen Doktorwũrde \\ der Hohen Philosophischen Fakultät der Albertus-Universitắt \\ zu Königsberg i. Pr. \\ vorgelegt von \\ Vera Bakšytè \\ aus Noreikiškiai (Litauen)
}

1930 - Vytauto Didžiojo - metai

Druck "Rytas"Aktien-Gesellschaft, Memel 


\section{LITUANIE \\ VERA BAKŠYTE, \\ LANGUE ET SURVIE \\ D'UN PEUPLE}

PAR PHILIPPE EDEL

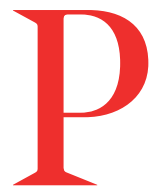

armi les documents relatifs à la Lituanie que possède la BNU figure la thèse de doctorat en philosophie de Vera Bakšyte, soutenue à l'université de Königsberg mais imprimée en Lituanie, à Klaipèda, en 1930.

Elle est consacrée à un épisode de l'histoire de la lutte pour la langue scolaire en Lituanie.

Le sujet de ce document est éminemment important pour les Lituaniens car il porte sur la sauvegarde de leur langue à l'époque où la majeure partie de leur pays était annexée à l'empire russe. La thèse relate cette lutte dans le cadre des grandes réformes engagées par le tsar Alexandre II durant la période 1855-1864. Parmi elles, la réforme de l'enseignement visait à relever le niveau de l'instruction dans l'empire en détachant l'enseignement primaire de la tutelle de l'Église, en créant des gymnases (lycées) ouverts théoriquement à tous les élèves sans distinction d'origine ou de religion, et en accordant une autonomie aux universités. Dans sa monographie, Vera Bakšytė s'attache cependant à mettre surtout l'accent sur l'éveil de la langue lituanienne et sa difficile émergence dans le système scolaire tsariste. Elle rappelle d'abord la place du lituanien dans la famille des langues indoeuropéennes et son importance dans la transmission orale de la culture et des traditions ancestrales et mythologiques lituaniennes par les chansons populaires («dainos»), les contes, les légendes et les proverbes. Elle retrace aussi l'évolution du statut de son enseignement durant la période antérieure, sous le grand-duché et lors de la réforme conduite par les jésuites à l'époque de la république des Deux Nations, l'influence de l'université de Vilnius sur les écoles qui lui étaient subordonnées ainsi que les conséquences de sa fermeture en 1832, l'impact des insurrections de 1830 et de 1863 et la politique de russification qui suivit. Elle décrit l'interdiction, introduite en 1864 par le pouvoir tsariste, d'imprimer livres et journaux lituaniens en caractères latins, et même plus tard en caractères gothiques, l'interdiction pour les enfants d'apprendre leur langue natale, suivie de la fermeture des écoles 
rurales, et l'interdiction d'utiliser le lituanien dans les administrations locales. Face à ces mesures restrictives, Vera Bakšytė rappelle le rôle qu'ont joué plusieurs personnalités, telles que Motiejus Valančius, évêque de Samogitie, qui encouragea les jeunes prêtres à écrire et à prêcher en lituanien et favorisa les publications clandestines ; Antanas Baranauskas, également prélat, auteur d'une des plus belles œuvres poétiques du $19^{\mathrm{e}}$ siècle et précurseur de la grammaire lituanienne ; Simonas Daukantas, premier historien à avoir écrit une histoire du pays en lituanien. L'interdiction de presse (« spaudos draudimas ») fut à l'origine des « knygnešiai " - littéralement porteurs ou passeurs de livres -, ces héros en Lituanie qui, pendant les quarante ans qu'a duré l'interdiction (1864-1905), ont assuré au risque de leur vie la survivance de la langue et de la culture lituaniennes en transportant sur leur dos livres et journaux qu'ils passaient clandestinement de Prusse orientale, de Tilsit et Ragnit où ils étaient imprimés, en Lituanie sous joug russe. On notera que la thèse s'appuie sur un appareil critique dense et une bibliographie composée essentiellement de sources en russe, polonais et lituanien, multipliant ainsi l'intérêt pour le lecteur ne maîtrisant pas ces langues.

Quand la thèse fut publiée en 1930, la Lituanie - redevenue indépendante au lendemain de la Première Guerre mondiale mais privée alors de sa capitale historique, Vilnius - entreprit un effort considérable pour développer l'instruction publique, eu égard à son retard par rapport à l'Europe occidentale. Suite aux restrictions de l'époque tsariste, un tiers de la population lituanienne, très majoritairement encore agricole, était en effet restée illettrée. Les dépenses du ministère de l'Instruction lituanien furent ainsi doublées entre 1923 et 1928, et le nombre des écoles primaires et secondaires, lycées, écoles professionnelles et agricoles tripla dans tout le pays durant les années 1920. Dans l'enseignement supérieur, l'université de Kaunas fut fondée en 1922, avec sept facultés, et l'Académie de l'agriculture à Dotnuva fut créée en 1924. Deux grandes bibliothèques, l'une nationale et l'autre universitaire, complétèrent le nouveau dispositif de l'enseignement et du savoir en Lituanie. Pour la première fois dans l'histoire du pays, tous ces établissements utilisaient exclusivement, comme langue d'enseignement, le lituanien qui venait opportunément, en ce début du $20^{\text {e }}$ siècle, d'être standardisé par le linguiste Jonas Jablonskis. Ainsi, grâce notamment aux efforts initiés par les pionniers décrits par Vera Bakšytè, le lituanien retrouvait par l'école sa capacité à assurer pleinement son rôle de support de communication pour la vie sociale, culturelle, économique et politique du pays.

Le destin de l'auteur de la thèse est aussi particulièrement intéressant car il est représentatif de celui d'une partie de l'élite lituanienne de son époque. Vera Bakšyté, connue plus tard sous son nom complet de Veronika Karveliené-Bakšytè, devint en effet un personnage public dans son pays. Née d'une famille paysanne dans le nord-est de la Lituanie, elle commença ses études secondaires de 1912 à 1915 au lycée Vinogradov de Vilnius, un de ces gymnases pour jeunes filles créés suite à la réforme étudiée dans sa thèse. Lors de la grande retraite russe de 1915, elle suivit sa famille à Voronej - à plus de $1000 \mathrm{kms} \mathrm{du}$ sud-est de la Lituanie - où elle acheva ses études au lycée lituanien de jeunes filles de la ville. Après avoir travaillé deux ans (1919-1921) à l'agence de presse lituanienne ELTA à Kaunas, capitale provisoire du pays redevenu indépendant, elle poursuivit des études de philosophie, philologie et pédagogie en Allemagne, dans les universités de Münster, Munich et Königsberg où elle soutint sa thèse de doctorat. De 1929 à 1940, elle vécut au domaine agricole de Noreikiškiai, près de Kaunas, que dirigeait son mari, Petras Karvelis, figure de la scène politique et économique lituanienne de l'entre-deux-guerres - il présida la Banque agricole de Lituanie et devint ministre des Finances. Elle créa une école populaire pour femmes et milita dans les organisations féminines catholiques dont elle devint la présidente. Puis éclata la Seconde Guerre mondiale. Lors de la première annexion soviétique de 1940, Vera Bakšytè se réfugia d'abord en Prusse. Avec le retour de l'Armée rouge en 1944, elle s'enfuit définitivement de Lituanie et connut le sort de la bonne centaine de milliers de "personnes déplacées ${ }^{1}$ originaires des pays baltes et réfugiées principalement dans le sud de l'Allemagne, dans la zone d'occupation française notamment. Accueillie d'abord à Tübingen, elle put s'établir en 1950 à Strasbourg où elle mourut en 1952, à l'âge de 54 ans. Sa pierre tombale au cimetière nord de la ville est l'œuvre d'un grand sculpteur lituanien, également en exil, Antanas Mončys. Composée d'une 
stèle ornée d'une piéta enserrant une croix, l'œuvre est si belle et originale qu'elle servit à illustrer la couverture du récent volume des guides des cimetières que la Ville de Strasbourg consacra à cette nécropole ${ }^{2}$. On notera que sa fille, Ugnè Karvelis, qui la suivit dans son exil (qu'elle décrit dans son roman Demain il n'y aura plus de trains $^{3}$ ), devint - après l'effondrement de l'URSS en 1991 - la première déléguée permanente auprès de l'UNESCO de la Lituanie à nouveau indépendante. C'est à ce titre qu'elle fut invitée à Strasbourg par la BNU en 1996, pour y faire une conférence sur la littérature lituanienne.

Pour conclure, signalons comme un double clin d'œil que c'est à Königsberg, la ville où Vera Bakšytè soutint sa thèse, que fut édité en 1547 le premier livre en lituanien ${ }^{4}$, et à Strasbourg, la ville de son dernier refuge, que fut imprimée en 1513 la première carte de l'Europe où il est fait mention de Vilnius, la capitale historique de la Lituanie $^{5}$.

\section{NOTES}

1- Displaced Persons - DPs, à l'origine de la création du Haut Commissariat des Nations Unies pour les réfugiés (UNHCR).

2- Strasbourg-Robertsau cimetière nord. Collection Guide des cimetières de la Ville de Strasbourg, ${ }^{\circ} 6$, Strasbourg, Communauté urbaine de Strasbourg, 2011

3- Karvelis, Ugnè, Demain il n'y aura plus de trains, Paris, La Différence, 1991

4- Mažvydas, Martynas / Masvidius, Martinus, Catechismvsa prasty szadei, makslas skaitima raschta yr giesmes, Königsberg, $\mathrm{H}$. Weinreich, 1547

5- Cusanus, Nicolaus, Tabula moderna Sarmatia Eur. (réédition de la Geographia de Claudius Ptolémée), Strasbourg, 1513 